\title{
Reciclagem do resíduo da serragem de calcário laminado para produção de blocos cerâmicos
}

\author{
Recycling of laminated calcite tile sawing waste for \\ the production of ceramic bricks
}

\section{Romualdo R. Menezes \\ Prof. Dr., Departamento de Engenharia de Materiais, UFPB. E-mail:romualdomenezes@gmail.com}

\section{Luiz Ronaldo L. Melo}

Prof. MSc., IFCE

E-mail: lisboa@cefet-ce.br

\section{Francisco Antonio Santos Fonsêca}

Aluno de Graduação, Unidade Acadêmica de Engenharia de Materiais, UFCG.

E-mail: toninhosf@gmail.com

\section{Pollyane M. Souto}

Pesquisadora Dr. Departamento de Engenharia de Materiais, UFSCar.

E-mail:pollyane@iris.ufscar.br

\section{Gelmires de A. Neves}

Prof. Dr., Unidade Acadêmica de Engenharia de Materiais, UFCG. E-mail:gelmires@dema.ufcg.edu.br

\section{Lisiane N. L. Santana}

Prof. Dr., Unidade Acadêmica de Engenharia de Materiais, UFCG. E-mail: lisiane@dema.ufcg.edu.br

\section{Resumo}

As indústrias da mineração e beneficiamento de calcário laminado representam um importante segmento econômico do Estado do Ceará, entretanto produzem uma grande quantidade de resíduos, que poluem e agridem o meio ambiente. Assim, esse trabalho tem por objetivo a caracterização do resíduo da serragem da Pedra Cariri e a avaliação de sua aplicabilidade como matéria-prima cerâmica alternativa para a produção de telhas e blocos cerâmicos. O resíduo foi caracterizado através da determinação de sua composição química e mineralógica, por difração de raios $\mathrm{X}$, análise térmica diferencial, distribuição de tamanho de partículas e análise morfológica por microscopia eletrônica de varredura. Foram formuladas composições contendo o resíduo e confeccionados corpos-de-prova por prensagem. Os corpos-de-prova foram queimados e, em seguida, foram determinados a absorção de água e o módulo de ruptura à flexão. Pode-se concluir que o resíduo é constituído por calcita e dolomita, que apresenta elevada finura e que é possível a incorporação de até $10 \%$ de resíduo em formulações para a produção de telhas e blocos cerâmicos.

Palavras-chave: Resíduo, reciclagem, pedra de calcário laminado, blocos cerâmicos, telhas.

\begin{abstract}
The mining and processing industries of laminated calcite tile are an important economic sector in the State of Ceará. However, they generate a large amount of wastes, which are a source of contamination and environmental pollution. This study aimed to characterize the laminated calcite tile sawing waste and evaluate its suitability as an alternative ceramic raw material for the production of bricks and roof tiles. The waste was characterized by chemical composition determination, $X$-ray diffraction, differential thermal analyses, particle size distribution determination, and morphological analysis by electronic scanning microscopy. Several formulations were prepared and sample bodies were prepared by uniaxial pressing. The sample bodies were fired at different temperatures. Sintered samples were characterized in terms of water absorption and mechanical strength. The results showed that the waste is composed of calcite and dolomite,
\end{abstract}


that presents a high level of fines. As such, it is possible to incorporate up to $10 \%$ for the production of ceramic bricks and roof tiles.

Keywords: Wastes, recycling, laminate calcite stone, bricks, roof tiles.

\section{Introdução}

Tradicionalmente, os resíduos são dispostos em aterros e, geralmente, descartados diretamente no meio ambiente. Os problemas ligados à poluição e ao descarte de resíduos vêm preocupando, tanto o setor industrial, quanto a sociedade como um todo. O simples descarte no meio ambiente é uma prática ilegal, que pode gerar uma série de danos ambientais e danos à saúde humana. Outro dado que torna a questão bastante complexa é o fato de que a disposição de resíduos, segundo as normas pertinentes, requer das empresas geradoras elevados investimentos (Menezes et al., 2002).

A inserção dos resíduos num ciclo produtivo representa uma opção de recuperação desses materiais, tornando tal ação em algo de interesse, tanto no aspecto ambiental, como no econômico (Andreola et al., 2002). Nesse sentido, a reciclagem destaca-se como uma das melhores alternativas para se minimizar um dos grandes problemas da sociedade moderna, a geração de resíduos.

Nos últimos anos, pesquisas sobre a reciclagem de resíduos industriais vêm se intensificando em todo o mundo. No entanto, ainda possui índices insignificantes frente ao montante produzido a cada dia (Bidone \& Povinelli, 1999). Tal fato evidencia que a busca de alternativas de reciclagem que conciliem as necessidades ambientais e o fator econômico é uma questão de suma importância para o crescimento econômico aliado ao desenvolvimento sustentável.

Cerâmicas tradicionais, como telhas, blocos e revestimento cerâmicos, geralmente, apresentam grande variação composicional, devido ao largo intervalo de composições das argilas utilizadas como matérias-primas para sua fabricação, o que possibilita uma grande tolerância para a incorporação de resíduos inorgânicos. O potencial de incorporação de resíduos, nas formulações de cerâmicas tradicionais, aliado as elevadas quantidades de recursos naturais consumidos a cada dia por esse segmento industrial, ressalta a importância da reutilização de resíduos como matérias-primas cerâmicas alternativas (Torres et al., 2004; Menezes et al., 2005; Raupp-Pereira et al., 2006).

Entre o diversificado universo de fontes poluidoras e geradoras de resíduos, têm-se as indústrias de processamento e beneficiamento mineral, cujo montante de resíduos produzidos caminha, paralelamente, com o aumento de produção que vem ocorrendo nos últimos anos. Em todo o mundo, milhões de toneladas de resíduos inorgânicos são produzidos a cada dia, nas atividades de mineração e de beneficiamento mineral, representando um enorme potencial de impactos ambientais (Menezes et al., 2007; Tiruta-Barna et al., 2007).

A indústria da extração e beneficiamento de rochas ornamentais de calcário laminado (conhecidas comercialmente como Pedra Cariri), utilizado no revestimento interno e externo de pisos, gera uma enorme quantidade de resíduos, tanto na usa extração, como no seu beneficiamento. Estima-se que as perdas na lavra variam de 60 a $90 \%$ do total extraído, conforme a operação seja com o uso de máquina com disco diamantado ou manual, respectivamente. Considerando-se toda a cadeia produtiva, etapas de lavra e beneficiamento, verifica-se uma perda média em torno de $70 \%$ do total extraído das jazidas (Vidal \& Padilha, 2003).

O grande desperdício, na produção de calcário laminado no Cariri Cearense, associado a mais de 30 anos de exploração e mineração com tecnologias inadequadas às condições das jazidas fizeram com que o volume de resíduos, só na região de Nova Olinda e Santana do Cariri (CE), pólos produtores desse material, atingisse, aproximadamente, 2,5 milhões de toneladas (Vidal et al., 2008).
Esses resíduos são, em geral, descartados indiscriminadamente em campo aberto, provocando danos ao meio ambiente e dificultando o avanço das frentes de lavra. Ademais, os efluentes líquidos gerados com o pó da serragem e com a calibração das rochas ornamentais provocaram o assoreamento dos leitos dos riachos existentes no entorno das serrarias e prejudicaram a cobertura do solo, danificando a vegetação.

Nos últimos anos, várias pesquisas têm sido dedicadas ao estudo da reutilização de resíduos da indústria da mineração e de beneficiamento de rochas ornamentais, com a finalidade de transformar tais resíduos em matériasprimas alternativas para a indústria de cerâmicas tradicionais (Campos et al., 2004; Vieira et al., 2004 Acchar et al., 2006; Torres et al., 2007; Menezes et al., 2008). No entanto, não se observam trabalhos analisando a potencialidade de reutilização do resíduo da serragem de calcário laminado para fins cerâmicos. Assim, esse trabalho tem, por objetivo, caracterizar o resíduo da serragem da Pedra Cariri e avaliar sua viabilidade para incorporação em massas cerâmicas, para a produção de blocos cerâmicos.

\section{Materiais e métodos}

Nessa pesquisa, foram utilizados os seguintes materiais: dois tipos de resíduos gerados na serragem da Pedra Cariri, denominados por "Amarelo" e "Cinza" (em virtude de sua coloração), provenientes de empresas do município de Nova Olinda e Santana do Cariri, CE, respectivamente; e argila vermelha. Os resíduos foram coletados, diretamente, nas empresas, após processo de quarteamento, e são representativos das duas "variedades" de Pedras Cariri produzidas na região. A argila vermelha foi estudada anteriormente (Almeida, 2006) e é constituída por caulinita, quartzo e mica. Apresenta uma larga distribuição de tamanho de partículas $\left(\mathrm{D}_{10}=2 \mu \mathrm{m} \mathrm{e}\right.$ $\left.\mathrm{D}_{90}=60 \mu \mathrm{m}\right)$, com tamanho médio de partícula de $8,22 \mu \mathrm{m}$. É utilizada para produção de blocos e de telhas pela Cerâmica Espírito Santo, PB. 
Romualdo R. Menezes et al.

Os resíduos foram caracterizados através da determinação da sua composição química, por fluorescência de raios X (Shimadzu EDX-90), difração de raios X (Siemens/Brucker, AXS D5005), análises térmica diferencial (ATD) (BP Engenharia, RB3000), determinação da distribuição do tamanho de partícula (Cilas, 1064LD) e microscopia eletrônica de varredura (MEV) (Phillips, XL30 FEG). Para os ensaios de caracterização, os resíduos foram cominuídos em almofariz e passados em peneira com abertura de $74 \mu \mathrm{m}$ (ABNT $\left.\mathrm{N}^{\circ} 200\right)$, a exceção do ensaio de determinação da distribuição granulométrica, para o qual os resíduos não foram cominuídos previamente.

Os resíduos foram secos a $100^{\circ} \mathrm{C}$, desaglomerados em moinho de bolas e peneirados através de peneira com abertura de $300 \mu \mathrm{m}\left(\mathrm{ABNT}^{\circ}{ }^{\circ} 50\right)$. Em seguida, foram preparadas formulações através da mistura a seco em moinho de bolas por $2 \mathrm{~h}$. As proporções de resíduos adicionados à argila vermelha foram de $5,10,15,20,25$ e $30 \%$. Após mistura em moinho de bolas, as formulações foram, novamente, passadas por peneira com abertura de $300 \mu \mathrm{m}\left(\mathrm{ABNT} \mathrm{N}^{\circ}\right.$ 50). Em seguida, foram confeccionados corpos-de-prova de $60 \mathrm{~mm}$ x $20 \mathrm{~mm}$ x 5 $\mathrm{mm}$ por prensagem uniaxial a $27 \mathrm{MPa}$. Tais corpos-de-prova foram queimados a 900,950 e $1000^{\circ} \mathrm{C}$.

Os corpos-de-prova queimados foram submetidos a ensaio de caracterização física, com determinação da absorção de água e módulo de ruptura a flexão (com velocidade de aplicação de carga de $0,5 \mathrm{~mm} / \mathrm{min}$ ). Os ensaios cerâmicos foram conduzidos segunda a metodologia de ensaios preliminares proposta por Barzaghi e Salge (1982) e Souza Santos (1992), para caracterização de matérias-primas para a indústria cerâmica.

\section{Resultados e discussão}

A Figura 1 apresenta as curvas de distribuição granulométrica dos resíduos estudados. Pode-se observar que os resíduos apresentam uma estreita distri- buição de tamanhos de partículas, com distribuição bimodal e concentração de partículas em torno de 2 e $8-10 \mu \mathrm{m}$. Verifica-se que $100 \%$ do material apresenta tamanho de partícula inferior a $25 \mu \mathrm{m}$, sendo o tamanho médio de partícula de 4,9 e $6,1 \mu \mathrm{m}$, para o resíduo amarelo e cinza, respectivamente. Verifica-se que os resíduos, amarelo e cinza, apresentam distribuições granulométricas similares, o que está relacionado às semelhanças de processamento (serragem) e de formação geológica do calcário beneficiado.

A Tabela 1 apresenta as composições químicas, semiquantitativas, dos resíduos estudados. Observa-se que os resíduos apresentam uma pequena diferença de composição química, com o resíduo cinza possuindo $\mathrm{MgO}$ em sua composição e o amarelo, não, o que é indicação da presença de dolomita ou de calcário magnesiano no resíduo cinza. Ambos os resíduos apresentaram pequenas contaminações com $\mathrm{SiO}_{2}$ e $\mathrm{Fe}_{2} \mathrm{O}_{3}$, entretanto o cinza apresentou um maior teor de ferro. Tal fato pode estar associado à coloração mais escura desse resíduo e, também, ao seu comportamento após queima.
A Figura 2 apresenta os difratogramas de raios $\mathrm{X}$ dos resíduos estudados. Observa-se que o resíduo amarelo apresenta, como fase cristalina, apenas a calcita, enquanto o resíduo cinza possui calcita e dolomita. Assim, o $\mathrm{MgO}$ observado na análise química do resíduo cinza está associado à presença de dolomita. Entretanto pode-se observar que, apesar da presença da dolomita, a fase majoritária é a calcita.

A Figura 3 apresenta as curvas de análise térmica diferencial dos resíduos estudados. Observa-se que curva de ATD do resíduo amarelo possui um pico endotérmico por volta de $940^{\circ} \mathrm{C}$, provavelmente associado à decomposição do carbonato de cálcio presente no resíduo. A curva do resíduo cinza apresenta dois picos endotérmicos, 720 e $940^{\circ} \mathrm{C}$, possivelmente relacionados à decomposição da dolomita e do carbonato de cálcio presentes no resíduo, respectivamente. Observa-se, também, uma leve banda exotérmica entre 250 e $500^{\circ} \mathrm{C}$, aproximadamente, e pequeno pico exotérmico por volta de $475^{\circ} \mathrm{C}$. Tanto o aparecimento da banda quanto o aparecimento do pico podem estar relacionados a queima de matéria orgânica.

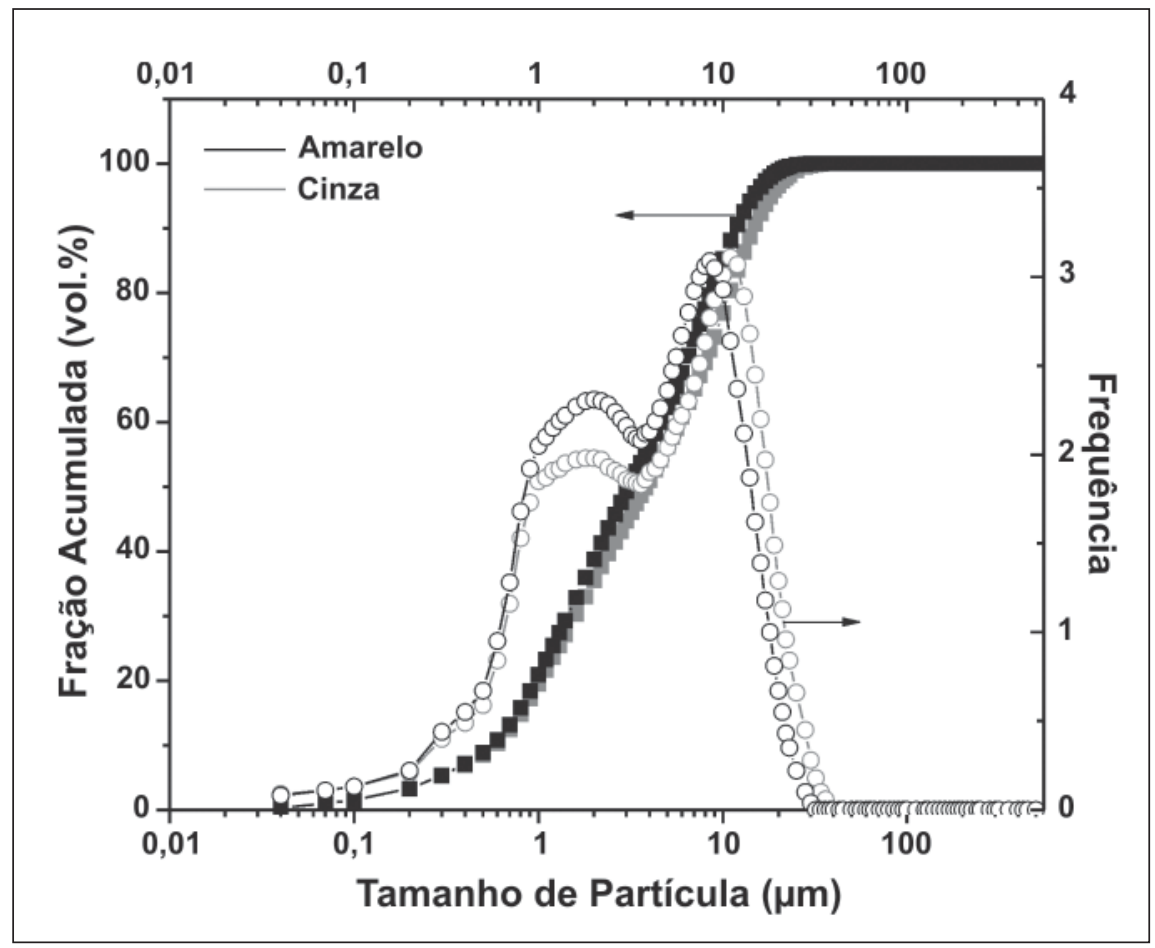

Figura 1 - Distribuições de tamanho de partícula dos resíduos da serragem de calcário laminado. 
Reciclagem do resíduo da serragem de calcário laminado para produção de blocos cerâmicos

Tabela 1 - Composição química dos resíduos estudados da serragem de calcário laminado.

\begin{tabular}{c|c|c|c|c|c|c|c|c|c}
\hline Amostras & $\mathrm{CaO}$ & $\mathrm{SiO}_{2}$ & $\mathrm{MgO}$ & $\mathrm{Fe}_{2} \mathrm{O}_{3}$ & $\mathrm{MnO}$ & $\mathrm{ZnO}$ & $\mathrm{SrO}$ & $\mathrm{CuO}$ & $\mathrm{PF}^{\mathrm{a}}$ \\
\hline Res. Amarelo & 54,39 & 0,85 & - & 0,66 & 0,22 & 0,03 & 0,09 & 0,01 & 43,74 \\
\hline Res. Cinza & 51,79 & 0,57 & 2,04 & 1,41 & 0,34 & 0,01 & 0,08 & 0,01 & 43,75 \\
\hline
\end{tabular}

${ }^{a}$ Perda ao Fogo.

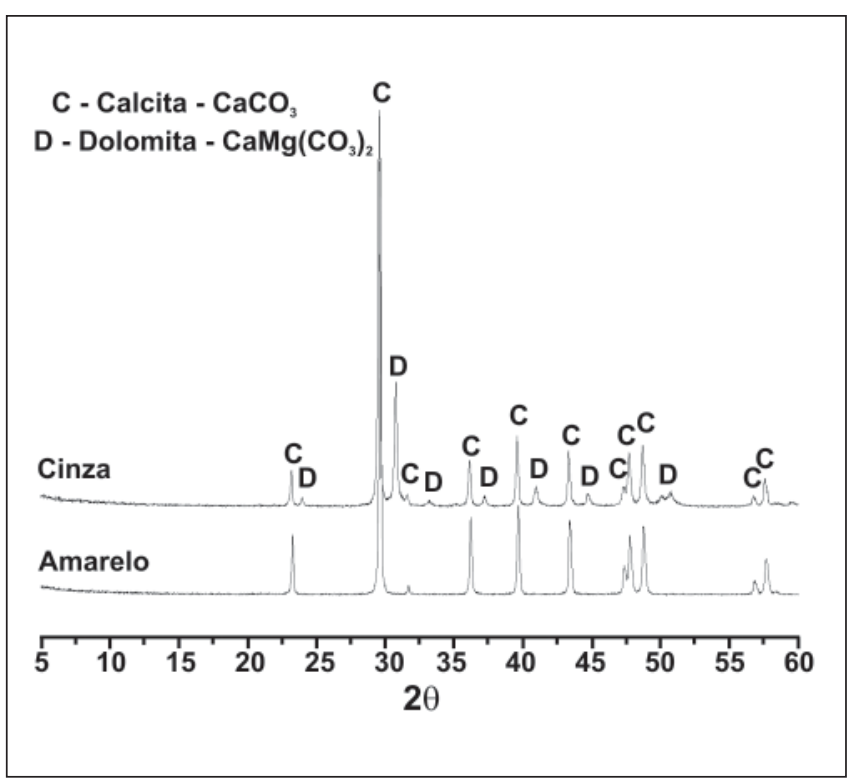

Figura 2 - Difratograma de raios $X$ dos resíduos da serragem de calcário laminado.

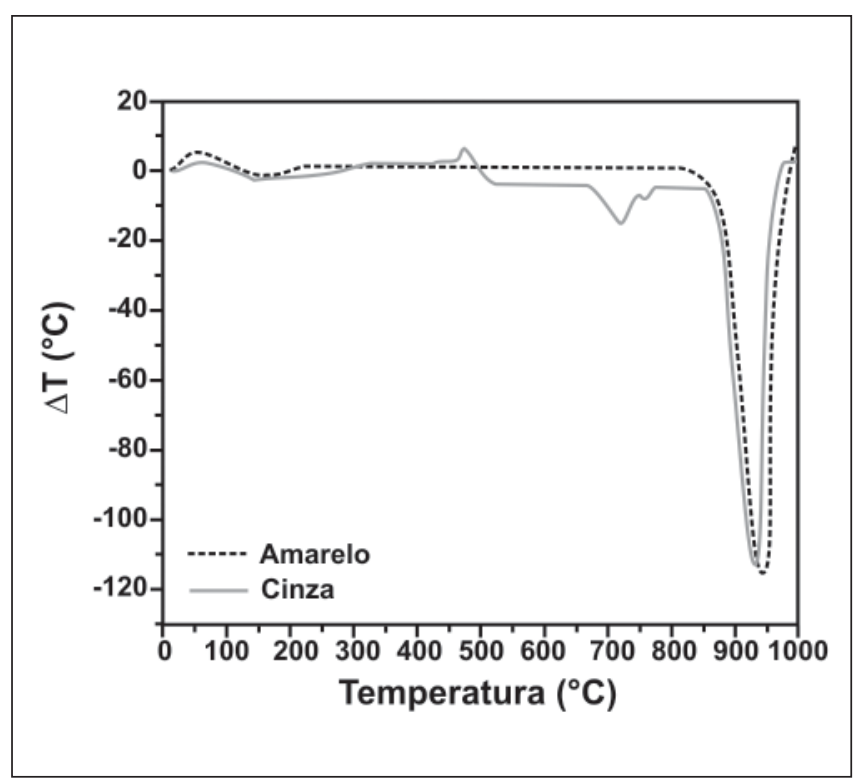

Figura 3 - Análise térmica diferencial dos resíduos da serragem de calcário laminado.
As Figuras 4a e 4b apresentam as micrografias eletrônicas dos resíduos. Através do ensaio de microscopia, verifica-se que as partículas dos resíduos com tamanho em torno de $1 \mu \mathrm{m}$ (observadas na Figura 1) são constituídas por aglomerados de partículas menores. Assim, o resíduo apresenta elevada finura, da ordem de submícrons. Observa-se, também, que as partículas menores apresentam uma morfologia lamelar, enquanto as maiores aparentam ser formadas por camadas, o que está associado à origem geológica desse material (estratificação de sedimentos).

A Figura 5 apresenta a absorção de água e o módulo de resistência a flexão dos corpos-de-prova produzidos com incorporações de resíduos. Com base na Figura 5, pode-se observar que, de forma geral, há uma tendência de aumento da absorção de água com a elevação do teor de resíduo incorporado. No entanto, observa-se que esse comportamento é um pouco distinto entre os resíduos. Nos corpos contendo resíduo cinza, há uma aumento gradual da absorção com a adição do resíduo, já, nos corpos contendo resíduo amarelo, inicialmente há uma diminuição da absorção seguidapor um aumento gradual até se terem absorções superiores ao da argila pura $(0 \%$ de resíduo). O resíduo cinza contém um pouco de dolomita, que se decompõe em torno de 650 e $700^{\circ} \mathrm{C}$, como observado na Figura 3. Assim, quando da queima dos corpos contendo o resíduo em temperaturas superiores a $900^{\circ} \mathrm{C}$, ocorre a decomposição total ou de grande parte desse componente, gerando gases e, por conseguinte, aumentando a porosidade e a absorção do material.

Por outro lado, o resíduo amarelo não possui dolomita e a decomposição da calcita inicia-se por volta de $850^{\circ} \mathrm{C}$. Assim, quando da adição de pequenas quantidades de resíduo, há a geração de gases em pequena quantidade, já que a temperatura de decomposição do resíduo é elevada, tendo esse fenômeno (quando da adição de pequenas quantidades) um efeito menor na porosidade do que na decomposição da dolomita. Durante a análise térmica, observou-se, através de termogravimetria, que, taxas de aquecimento de $10^{\circ} \mathrm{C} / \mathrm{min}$ eram demasiadamente rápidas para provocarem a total decomposição da calcita (essa análise foi conduzida utilizando elevada quantidade de amostra e não amostra em miligramas), o que vem ao encontro do hipótese levantada. Juntamente com a menor geração de gases, quando da queima dos corpos contendo resíduo amarelo, tem-se que o resíduo apresenta elevada finura, Figura 1, o que indica que pode atuar como enchimento da massa cerâmica, diminuindo a porosidade do corpo após queima. Isto está de acordo com o observado na absorção dos corpos contendo pequenas quantidades de resíduo. Quando se utilizam maiores quanti- 


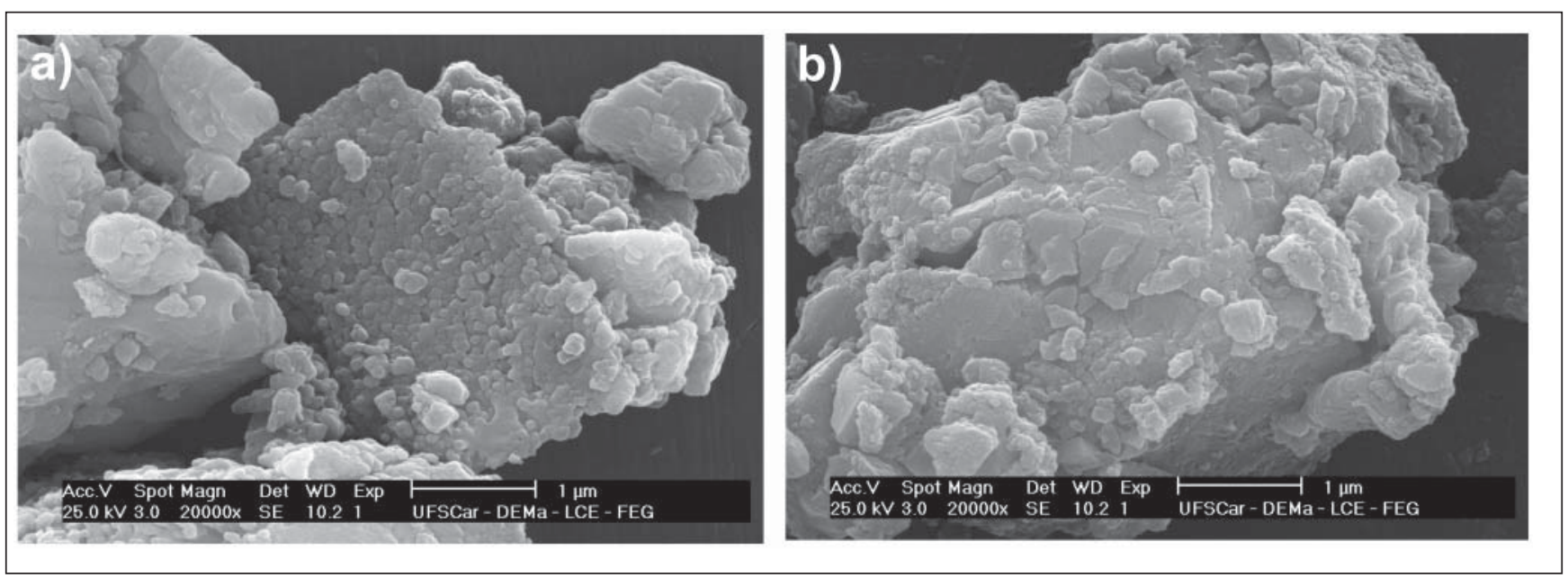

Figura 4 - Micrografias de microscopia eletrônica de varredura dos resíduos da serragem de calcário laminado: a) amarelo, b) cinza.

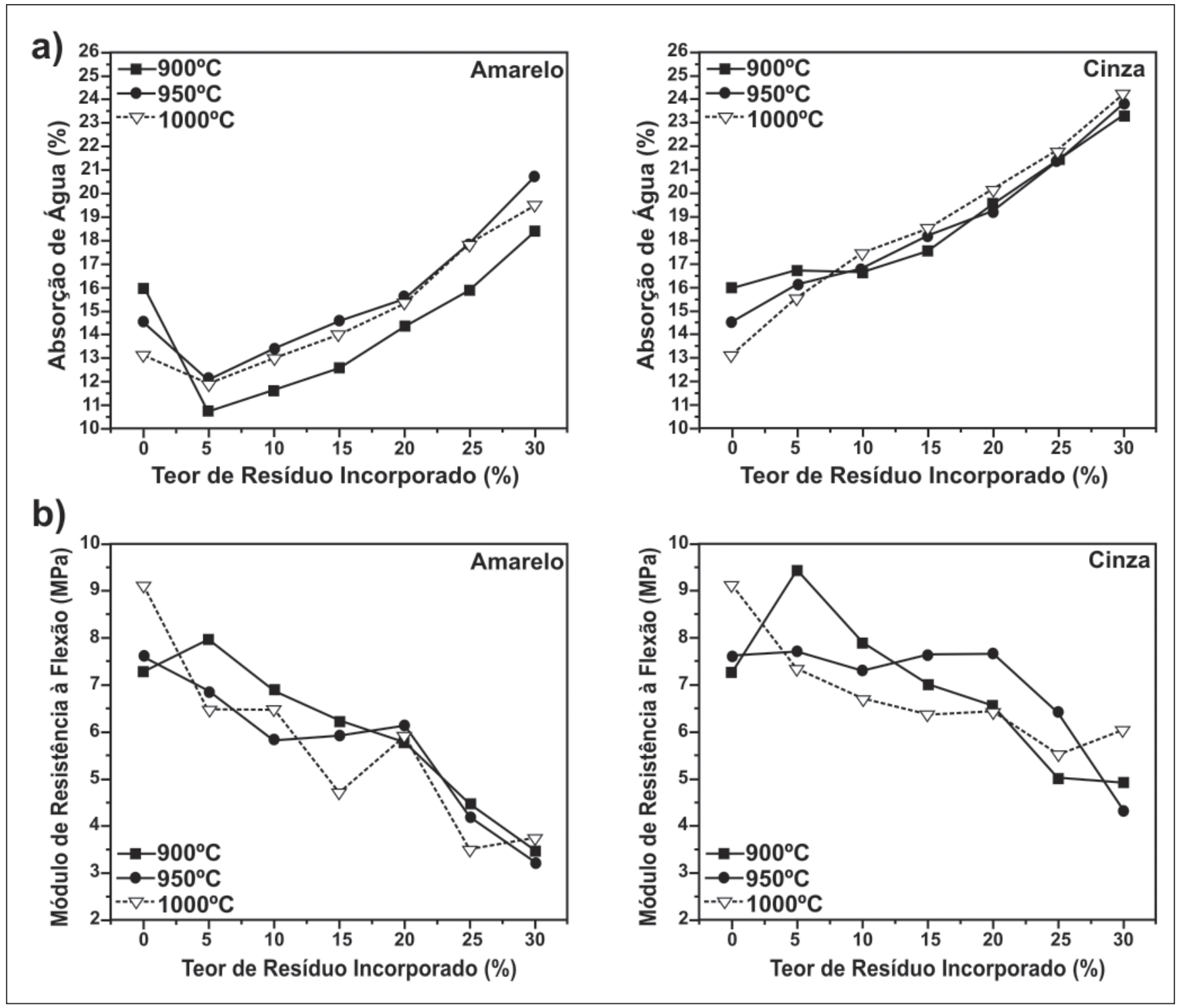

Figura 5 - Absorção de água (a) e módulo de ruptura à flexão (b) dos corpos-de-prova contendo resíduos de calcário laminado. 
dades, superiores a $20 \%$, observa-se que o efeito de emissão de gases é predominante e há um aumento da absorção, em relação ao material sem resíduo.

O módulo de ruptura dos corpos contendo resíduos apresentou uma tendência geral de decréscimo com o aumento de resíduo incorporado, independentemente de qual o resíduo utilizado. Isso é interessante porque indica que, apesar da diminuição da absorção, observada quando da adição de pequenas quantidades de resíduo, o efeito da emissão de gases, durante a queima, é superior a um melhor empacotamento do sistema, conduzindo a uma diminuição da resistência do material. A geração de gases pode gerar trincas e fissuras, que, apesar de não alterar a absorção do material, podem comprometer, significativamente, sua resistência, tal como observado. No entanto, é importante destacar o aumento de resistência observado, quando da queima a $900^{\circ} \mathrm{C}$ dos corpos-de-prova contendo $5 \%$ de resíduo (tanto amarelo quanto cinza), o que pode estar associado ao que já foi mencionado anteriormente, ou seja, a pequena emissão de gases, quando da queima nessa temperatura, faz com que o efeito de empacotamento seja o efeito mais importante na determinação da resistência. Deve-se destacar esse resultado porque a absoluta maioria das olarias da região nordeste, incluindo as encontradas nos arredores da região mineradora, utilizam temperaturas de queima entre 850 e $900^{\circ} \mathrm{C}$, o que evidencia um enorme potencial de utilização do resíduo, já que não se atinge, ou atingi-se levemente, a temperatura de decomposição do carbonato, fazendo com que apenas o lado benéfico de sua utilização tenha ação e possa-se incorporar o resíduo na produção de blocos sem deterioração das propriedades mecânicas do material.

Com base em indicações da literatura (Souza Santos, 1992), observa-se que as formulações contendo até $15 \%$ de resíduos, sejam amarelos ou cinzas, podem ser utilizadas em cerâmica vermelha. Conforme literatura (Barzaghi \& Salge, 1982), as formulações com até
$10 \%$ de resíduos podem ser utilizadas para a produção de blocos maciços, furados e telhas (módulo de ruptura mínimo de 2,0, 5,5 e 6,5 MPa, respectivamente), mesmo após queima a $900^{\circ} \mathrm{C}$.

\section{Conclusão}

Considerando que esse trabalho teve, por objetivo, a caracterização de resíduos da serragem da Pedra Cariri e a avaliação de sua aplicabilidade como matéria-prima cerâmica alternativa, para a produção de blocos cerâmicos, podese concluir, com base nos resultados obtidos, que os resíduos são constituídos por calcita e dolomita, que apresentam estreita distribuição de tamanho de partícula e elevada finura, com tamanho médio de partícula em torno de $5 \mu \mathrm{m}$; que seus constituintes se apresentam em elevado estado de aglomeração; que, de forma geral, a absorção de água dos corpos-de-prova contendo resíduo aumenta e o módulo de ruptura diminui com a elevação da quantidade de resíduo, independentemente da temperatura de queima; que é possível a incorporação de até $10 \%$ de resíduo em formulações para produção de tijolos maciços e furados e telhas.

\section{Agradecimentos}

Os autores agradecem ao $\mathrm{CNPq}$ (475010/2007-8) e à FAPESQ/MCT/ CNPq (003/03 e 004/06) pelo apoio financeiro.

\section{Referências bibliográficas}

ACCHAR, W., VIEIRA, F. A., SEGADÃES, A. M. Using ornamental stone cutting rejects as raw materials for red clay ceramic products: Properties and microstructure development. Materials Science and Engineering A, v. 435-436, p. 606-610, 2006.

ALMEIDA, R.R. Reciclagem de resíduo de caulim e granito para produção de blocos e telhas. Campina Grande-Paraíba: Universidade Federal de Campina Grande, 2006. 160 p. (Dissertação de Mestrado).

ANDREOLA, F. et al. Utilisation of municipal incinerator grate slag for manufacturing porcelainized stoneware tiles manufacturing.
Journal of the European Ceramic Society, v. 22 , p. 1457-1462, 2002.

BARZAGHI, L., SALGE, A. Resistência àflexão e moldagem de argilas. Cerâmica, v. 28, n. 151, p. 15-17, 1982.

BIDONE, F. R. A., POVINELLI, J. Conceitos básicos de resíduos sólidos. São Carlos: EESC-USP, 1999. 124p.

CAMPOS, M. et al. Recovered slate waste as raw material for manufacturing sintered structural tiles. Journal of the European Ceramic Society, v. 24, p. 811-819, 2004.

MENEZES, R. R., NEVES, G. A., FERREIRA, H. C. O estado da arte sobre o uso de resíduos como matérias-primas cerâmicas alternativas. Revista Brasileira de Engenharia Agrícola e Ambiental, v.6, n.2, p.303-313, 2002.

MENEZES, R. R. et al. Use of granite sawing wastes in the production of ceramic bricks and tiles. Journal of the European Ceramic Society, v. 25, p. 1149-1158, 2005.

MENEZES, R. R. et al. Análise da co-utilização do resíduo do beneficiamento do caulim e serragem de granito para produção de blocos e telhas cerâmicos. Cerâmica, v. 53, p. 192-199, 2007.

MENEZES, R. R. et al. Optimization of wastes content in ceramic tiles using statistical design of mixture experiments. Journal of the European Ceramic Society, v. 28, p. 3027-3039, 2008.

RAUPP-PEREIRA, F. et al. Ceramic formulations prepared with industrial wastes and natural sub-products. Ceramics International, v. 32, p. 173-179, 2006.

SOUZA SANTOS, P. Ciência e tecnologia de argilas. (3. Ed.). São Paulo: Edgard Blücher, 1992, v. 1. 408p.

TIRUTA-BARNA, L., BENETTO, E., PERRODIN, Y. Environmental impact and risk assessment of mineral wastes reuse strategies: review and critical analysis of approaches and applications. Resources, Conservation and Recycling, v. 50, n. 4, p. 351-379, 2007.

TORRES, P. et al. Incorporation of granite cutting sludge in industrial porcelain tile formulations. Journal of the European Ceramic Society, v. 24, p. 3177-3185, 2004.

TORRES, P. et al. Development of ceramic floor tile compositions based on quartzite and granite sludges. Journal of the European Ceramic Society, v. 27, p. 4649-4655, 2007.

VIDAL, F. W. H., PADILHA M. W. M. A indústria extrativa da pedra cariri no estado do Ceará. In: SIMPÓSIO DE ROCHAS ORNAMENTAIS DO NORDESTE, 4. Anais... Ceará, 2003. p. 199-210.

VIDAL, F. W. H., PADILHA, M. W. M., OLIVEIRA, R. R. Aspectos do aproveitamento dos rejeitos da Pedra Cariri. Centro de tecnologia Mineral. Disponível em: http://www.cetem.gov.br/publicacao/CTs/ CT2005-013-00.pdf. Acesso em 4 jan. 2008.

VIEIRA, C. M. F. et al. Incorporation of granite waste in red ceramics. Materials Science and Engineering A, v. 373, p. 115-121, 2004.

Artigo recebido em 08/09/2009 e aprovado em 28/01/2010. 